\section{The effect of category cues on the free recall of retarded adults}

\author{
PETER HERRIOT* \\ Hester Adrian Research Centre \\ Manchester University, Manchester 13, England
}

Categorically related items were presented in a free recall task simultaneously by slide projection to 80 retarded adults. Category cues were provided at presentation, at recall, or at both presentation and recall: clustering increased as a result of any $E$ intervention, even when recall cues signaled different categories from presentation cues; recall, on the other hand, increased only when the same presentation and recall cues were provided. It was concluded that an increase in recall requires a hierarchical coding strategy and that retarded adults are capable of such a strategy.

The relation between organization and recall may not be as clear as has been supposed (Puff, 1970). It is possible that organization results in increased recall only when it is hierarchical in nature. That is, the load on capacity is decreased only when items are stored under superordinate codings and retrieved thereby. Clustering, on the other hand, could be increased as the result of a sequential organizational strategy; each item could be used to cue the next and would be more likely to cue a categorically related than a categorically unrelated item. Thus, while both sequential and hierarchical organizational strategies might result in increased clustering, only a strategy with a hierarchical component is likely to lead to increased recall

The free recall task administered to retardates has provided further evidence of the differences between organization and amount recalled. Gerjouy \& Winters (1970) speculate on passible explanations for the absence of positive correlations between clustering and recall in their own and previous work. They favor an explanation in terms of modality-specific limitations. However, if the hypothesis outlined above is correct, the provision of superordinate codings in the form of cues by $E$ should result in increased recall in certain situations. Specifically, if information-processing capacity in the retarded is as limited as Olson (1971) suggests, cues should be provided at both presentation and recall if $E$ is to ensure that $S$ uses superordinate codings for both storage

*This research was carried out while the author was in receipt of a grant from the Leverhulme Trust. He wishes to thank Josephine Green and Roy McConkey for their help in conducting the experiment and the managers. staff, and trainees of Wythenshawe and Blackley Adult Training Centres, Manchester, England, for their kind cooperation. and retrieval. For cues provided at presentation alone might not be retained; cues provided at recall alone might not reinstate codings applied by the $\mathrm{S}$ himself at presentation. Tulving \& Osler (1968) established the superiority of cues at both presentation and recall, although they used one cue per item. Clustering, on the other hand, should not require both presentation and recall cues; the signaling of interitem relations at presentation or instructions at recall to emit items in categorical order should result in increased clustering by themselves. Indeed, if the ordering of recall can be independent of ordering at presentation, instructions to recall in a different order from that in which material was presented should still result in increased clustering.

The primary aim of the present experiment was to show that superordinate cues provided at both presentation and recall can increase amount recalled by adult retardates; previous failures to obtain effects on recall when pictorial presentation was employed may then be attributed to ineffective cueing rather than to a limitation of hierarchical processes to the auditory modality

\section{SUBJECTS}

The Ss were 80 retarded adults attending day adult training centers. They were allocated 8 to a group in such a way that groups were approximately equated on vocabulary age (VA) as measured by the English Picture Vocabulary Test, chronological age (CA), and basic recall ability (BRA). Basic recall ability was assessed by the number of 16 unrelated items correctly recalled within $80 \mathrm{sec}$ after one 80-sec presentation trial, presentation being pictorial and simultaneous. Mean scores for groups ranged from 7.30 (SD 1.33) to 7.81 (SD 0.71) on VA; from 22.0 (SD 2.32) to 26.3 (SD 8.26) on $\mathrm{CA}$; and from 7.88 (SD 2.42) to 9.00 (SD 1.41) on BRA. All Ss could point to the colors red, green, blue, and yellow when requested.

\section{MATERIAL}

There were 16 items from four categories. They were all manufactured objects (transport, toys, furniture, and clothes). Therefore, the color of the items was relatively arbitrary. Two sets of material were constructed; in the first, all the items from the same category were of the same color, and, in the second, every item within each category was a different color. Each item was surrounded by flashes of its own color in order to make the color feature more noticeable. The 16 items were randomly arranged in a 4 by 4 matrix; four different arrangements were prepared, one for each presentation trial and one for a preliminary naming trial. These arrangements were photographed onto slides, which were presented in the same order to all Ss by means of a Kodak Carousel projector.

\section{DESIGN AND PROCEDURE}

The free recall task consisted of three consecutive 60 -sec presentation trials immediately followed by three consecutive recall trials of the same duration. In this way, the effects of the provision of codings at presentation and recall can be distinguished better than they can in the usual multitrial free recall procedure.

There was thus a three-factor design with repeated measures on the third factor. The factors were sets of material, conditions, and trials. There were 10 groups, with five conditions for each of the two sets of material. The five conditions were no cues (N), category cues at presentation only (P), category cues at recall only (R), category cues at both presentation and recall (PR), and color cues at presentation followed by category cues at recall (CPR). Clearly, in the set where the color and category cues are compatible (i.e., when the items from a given category are all of the same color), the presentation and recall cues in the CPR condition refer to the same group of items. However, where they are incompatible, color cues at presentation will refer to a different group of items from the category cues at recall.

Procedure for the two $\mathrm{N}$ conditions was as follows. The $E$ told $S$ that he would show him some pictures a few times, than take them away, and $\mathbf{S}$ would have to tell him what they were. This instruction was repeated. During each presentation trial, E pointed to each item in a random order, and required $S$ to name it. In the two $P$ conditions, $U$ uttered a category cue, then pointed to the four appropriate category items, requiring $S$ 
Table 1

Mean Recall Pooled Over Trials for All Groups

\begin{tabular}{lccccr}
\hline & \multicolumn{5}{c}{ Condition } \\
\cline { 2 - 5 } & $\mathrm{N}$ & $\mathrm{P}$ & $\mathrm{R}$ & $\mathrm{CPR}$ & PR \\
\hline & & & Compatible Set & & \\
Mean & 9.71 & 10.1 & 10.3 & 11.0 & 11.9 \\
SD & 2.26 & 2.92 & 1.93 & 2.84 & 3.67 \\
& & & Incompatible Set & & \\
Mean & 9.46 & 10.5 & $\mathbf{8 . 9 2}$ & 9.75 & 13.5 \\
SD & 2.25 & 3.66 & 3.12 & 2.21 & 2.77 \\
\hline
\end{tabular}

to name them. In the case of the $R$ conditions, $\mathrm{E}$ asked at recall, e.g., "What furniture was there?" When S ceased recalling furniture items, $E$ uttered another category cue; if $S$ had uttered a nonfurniture item, e.g., doll, the cue would have been appropriate to this item, e.g., "Yes, and what other toys were there?" In the case of the $P R$ conditions, the $P$ and $R$ procedures both applied. In the CPR conditions, $E$ uttered the color cues at presentation rather than the category cues. Before all conditions, $S$ received a familiarization trial in which it was ensured that he knew the names of the items.

\section{RESULTS AND DISCUSSION}

Table 1 presents mean recall scores for all groups pooled over trials. Analysis of variance resulted in only one significant effect, conditions $[F,(4,70)=3.57, \mathrm{p}<.02]$. Neither sets nor recall trials had significant effects. Analysis of group means, pooled over sets and trials, by Duncan's multiple range test showed that PR differed from all other conditions. Specifically, $\mathbf{N}<\mathbf{P R}$, $\mathrm{R}<\mathrm{PR} \quad$ (both $\mathrm{p}<.005$ ), $\quad \mathrm{P}<\mathrm{PR}$ $(\mathrm{p}<.01)$, and CPR $<$ PR $(\mathrm{p}<.05)$.

Category clustering scores were also calculated, using three measures: Bousfield \& Bousfield's (1966) O-E measure, in which recall and clustering are not entirely statistically independent since amount recalled places an upper limit on amount of clustering possible; Roenker, Thompson, \& Brown's (1971) $\mathrm{O}-\mathrm{E} / \mathrm{Max}-\mathrm{E}$ percentage measure, in which recall and clustering are totally independent; and Frankel \& Cole's (1971) $\mathrm{Z}$ scores, based on the runs test, which also claims to be independent of recall. Again analyses of variance revealed, for all three measures, a significant conditions effect. For the O-E measure, $F(4,70)=$ $14.7, \mathrm{p}<.001$; for the percentage measure, $F(4,70)=23.4, p<.001$; and, for the $Z$ measure, $F(4,70)=$ $11.6, p<.001$. Further analysis by Duncan's test revealed the following differences at the $p<.01$ level for all three measures of clustering between group means pooled over sets and trials: $\quad \mathbf{N}<\mathrm{PR}, \quad \mathrm{P}<\mathrm{PR}, \quad \mathrm{N}<\mathrm{CPR}$, $\mathrm{N}<\mathrm{R}$. In addition, for the $\mathrm{O}-\mathrm{E}$ and percentage measures, $\mathbf{N}<\mathbf{P}$; for the O-E measure, $R<P R$; and, for the percentage measure, $P<R$ and $\mathrm{P}<\mathrm{CPR}$.

In addition to the conditions effect, there was also a trials effect for the O-E measure only $[F(2,140)=4.25$, $p<.02]$. Further analysis by Duncan's test showed that Trial $1<$ Trial $2(p<.05)$ and Trial $1<$ Trial 3 (p $<.01)$. the three measures and recall were, for the O-E measure, 0.79 ; for the percentage measure, 0.33 ; and for the $\mathrm{Z}$ measure, 0.61 . Correlations over all scores between the three measures themselves were as follows: $\mathrm{O}-\mathrm{E}$ and percentage measures, 0.73 ; O-E and $\mathrm{Z}$ measures, 0.86 ; percentage and $Z$ measures, 0.74

These results suggest that, if organization is to result in improved recall in retarded adults, it has to be hierarchical in nature; that is, superordinate codings have to be applied to the material for storage purposes, retained, and employed for the retrieval of items. Thus, the only condition that results in a significant increase in recall over the $\mathrm{N}$ control condition was the PR condition, which assisted at both storage and retrieval. The PR condition was significantly more effective than were all other conditions: than $P$, indicating the importance of retaining codings; than $R$, indicating the importance of applying them at presentation; and than CPR, indicating the importance of the identity of codings and categories throughout the recall task.

In the case of clustering, however, results show that any $\mathrm{E}$ intervention can result in a more clustered order of recall than occurs in the $\mathrm{N}$ condition. $P, R$, and CPR, which have no effect on recall, do increase clustering ( $P$ only in the case of two of the three measures at the $p<.01$ level). Moreover, there is little evidence of differences between $R, P R$, and CPR. In only one measure (the $\mathrm{O}-\mathrm{E}$ measure, which is statistically related to recall) is $\mathbf{R}<\mathbf{P R}$ at the $<.01$ level. This absence of difference suggests that clustering in recall does not require the same codings to be used at presentation and recall.
Correlations over all scores between
Perhaps the most interesting result, however, is that category clustering may result from codings provided at recall, even when both the codings and the items to which they refer were different at presentation. In the CPR condition with incompatible material, mean clustering scores were, for the $\mathrm{O}-\mathrm{E}$ measure, 3.47 (SD 2.05), for the percentage measure, 81.0 (SD 28.1), and, for the $\mathrm{Z}$ measure, 2.47 (SD 1.82). In the same condition with compatible material, $\mathrm{O}-\mathrm{E}=4.90$ (SD 2.33), percentage measure $=87.9$ $(\mathrm{SD} 30.2)$, and $\mathrm{Z}$ measure $=2.66$ (SD 3.77). Clearly, neither the groupings nor the codings of presentation need have a significant effect upon the order of recall if codings are provided at recall.

Failure to obtain any effect of sets on recall or clustering is of interest. It suggests that semantic features of the material are employed by these Ss, rather than incidental perceptual features, at least in the case where Ss are forced to name the items at presentation.

With regard to the specific area of retardation, the results indicate that, when codings are provided at presentation and reinstated at recall, recall can improve over a control condition. However, it need not be inferred that these Ss are incapable of improved recall unless provided with codings at both presentation and recall. It may be, for example, that they are not aware that category organization is a profitable strategy for use in memorizing material. Results do suggest that the capacity of retarded adults to use organization to aid recall is not limited to auditory presentation only.

\section{REFERENCES}

BOUSFIELD, A. K., \& BOUSFIELD, W. A Measurement of clustering and of sequential constancies in repeated free recall. Psychological Reports, 1966, 19, 935-942.

FRANKEL, F., \& COLE, M. Measures of category clustering in free recall. Psychological Bulletin, 1971, 76, 39-44.

GERJOUY I R., \& WINTERS, J. J. Subjective organization by EMR adolescents in free recall: Bimodal presentation. American Journal of Mental Deficiency, 1970, 74, 509-516.

OL SON, D. R. Information-processing limitations of mentally retarded children. American Journal of Mental Deficiency, $1971,75,478.486$.

PUFF, C. R. Role of clustering in free recall. Journal of Experimental Psychology, $1970,86,384-386$.

ROENKER, D. L. THOMPSON, C. P., \& BROWN, S. C. Comparison of measures for the estimation of clustering in free recall. Psychological Bulletin, 1971, 76, 45-48.

TULVING, E, \& OSLER, S. Effectiveness of retrieval cues in memory for words. Journal of Experimental Psychology, $1968,77,593-601$. 